\title{
Lipid composition of hamster epididymal spermatozoa
}

\author{
M. Awano ${ }^{1}$, A. Kawaguchi ${ }^{1}$ and H. Mohri ${ }^{2 *}$ \\ ${ }^{1}$ Department of Biology, College of Arts and Sciences, the University of Tokyo, Komaba, Meguro-ku, \\ Tokyo 153; and 'University of the Air, Wakaba, Mihama-ku, Chiba 261, Japan
}

\begin{abstract}
The lipid composition of hamster epididymal spermatozoa was examined. Caput epididymal spermatozoa were isolated by Percoll density gradient centrifugation without contamination by other cells and they had a specific gravity of $1.10-1.12 \mathrm{~g} \mathrm{~cm}^{-3}$. Caput and cauda epididymal spermatozoa showed little difference in the amounts of total fatty acid and total sterol. However, sterol composition changed markedly during the transit of spermatozoa through the epididymis: the amount of cholesterol decreased, while the amount of desmosterol and cholesta-7,24-dien-3 $\beta$-ol increased. No significant change in fatty acid composition was observed during the transit, although there was a tendency for an increase in chain length. Both 22:5 and 22:6 represented high percentages in fatty acids of hamster spermatozoa. Some difference in lipids was detected between the upper fraction $\left(1.04 \mathrm{~g} \mathrm{~cm}^{-3}\right)$ and the lower fraction ( $1.10 \mathrm{~g} \mathrm{~cm}^{-3}$ ) obtained by the density gradient centrifugation of cauda epididymal spermatozoa. Total fatty acid content of the upper fraction was 1.4-fold higher than that of the lower fraction, and the percentage of 18:0 was lower in the latter fraction with a higher percentage of 18:2. The total sterol:total phospholipid ratio in hamster cauda epididymal spermatozoa was 0.21 .
\end{abstract}

\section{Introduction}

During the transit through the epididymis after spermiation, mammalian spermatozoa gradually acquire progressive motility and fertilizing ability. This process is known as epididymal maturation. Various structural and functional changes occur in spermatozoa during epididymal maturation (Austin, 1985). Thus, loss of total and individual proteins (RodriguezMartinez et al., 1990), increased or decreased concentration of cAMP (Amann et al., 1982; White and Aitken, 1989), changes in cAMP-dependent protein kinase activity (Atherton et al, 1985; Wooten et al., 1986), changes in the pattern of glucose degradation and in the capacity for protein synthesis (Cornwall et al., 1990) have been observed. Dacheux et al. (1989) and Schlegel et al. (1986) reported that changes occur in the sperm plasma membrane during epididymal maturation, and there is abundant evidence that these changes are due partly to extracellular luminal fluid proteins synthesized and secreted by the epididymis. An increase in the density of negatively charged residues on the surface of mammalian spermatozoa is found during epididymal transit (Bedford, 1963; Cooper and Bedford, 1971) and changes in distribution and density of lectin binding sites were reported by Lui et al. (1991) and Magargee et al. (1988). The lipid content of spermatozoa decreases during epididymal maturation in bulls (Poulos et al., 1973), rams (Poulos et al., 1975; Evans and Setchell, 1979b), rats (Adams and Johnson, 1977) and boars

*Correspondence.

Received 23 October 1992
(Evans and Setchell, 1979a), although an accumulation of choline plasmalogen was noted in rabbits (Teichman ef al., 1974). Thus, the utilization of lipids as energy sources during the transit through the epididymis has been postulated. Changes in composition of sperm lipids have been reported by Nikolopoulou et al. (1985). Such changes in the composition and content of lipids in spermatozoa during epididymal maturation would cause the difference in susceptibility to cold shock (Hammerstedt et al., 1979) and in membrane fluidity (Vijayasarathy et al., 1979; Wolf and Voglmayr, 1984) between testicular spermatozoa and ejaculated spermatozoa. These phenomena seem to involve the plasma membrane. It is necessary to analyse lipids of whole spermatozoa to evaluate the relation between changes in spermatozoa during maturation and the plasma membrane, because lipid is one of the main components of the plasma membrane. However, there are a few reports on the change in the density (specific gravity) of mammalian spermatozoa during maturation in the epididymis by, for example, Lavon et al. (1966). Oshio (1988) reported that profiles of sperm distribution after Percoll density gradient centrifugation showed two separated main peaks in hamster cauda epididymal spermatozoa as well as in those of several other species. He postulated that this feature in sperm distribution profiles is due mainly to differences in the degree of maturity of spermatozoa.

In the present study, the lipid composition of hamster spermatozoa from caput and cauda epididymides were examined to detect changes during sperm maturation. Quantitative and qualitative analyses were also made with the lipids from the two fractions of cauda epididymal spermatozoa obtained by Percoll density gradient centrifugation. 


\section{Materials and Methods}

\section{Animals}

Two-month-old male hamsters (Mesocricetus auratus) were used. Animals (200-250 g body weight) were raised and maintained in an air-conditioned room with a $14 \mathrm{~h}$ light:10 h dark photoperiod at $23-25^{\circ} \mathrm{C}$.

\section{Chemicals}

Percoll and colour density marker beads were purchased from Pharmacia Fine Chemicals (Uppsala); BSA (Fraction V) was obtained from Sigma Chemical Co. (St Louis, MO). Phospholipid standards, docosaenoic acid (22:0), and $5 \alpha$-cholestane were purchased from Seikagaku Kogyo Co. (Tokyo). All solvents and other chemicals used were of analytical reagent grade.

\section{Preparation of sperm suspension}

Animals were killed by cervical dislocation. The epididymides were dissected into their caput and cauda regions. Spermatozoa were obtained from these regions by puncturing. Cauda epididymal spermatozoa were washed twice with PBS, $\mathrm{pH} 7.4$, to eliminate the epididymal fluid and centrifuged at $600 \mathrm{~g}$ for $10 \mathrm{~min}$ at room temperature. The washed cauda epididymal spermatozoa were used as a control (designated as unseparated fraction). For Percoll density gradient centrifugation, unwashed cauda epididymal spermatozoa (about $1 \times$ $10^{8}$ cells) were suspended in $10 \mathrm{ml}$ of modified Tyrode's solution (Bavister, 1969) containing 3\%(w/v) BSA, pH 7.4. Spermatozoa were dispersed in the medium for 15-20 min. Caput epididymal spermatozoa were suspended in a small volume of Tyrode's solution containing 3\% BSA. The number of caput epididymal spermatozoa from an individual was so small (about $1 \times 10^{6}$ cells) that samples were pooled and combined. Spermatozoa from ten caput epididymides were used for single lipid analysis. All operations were carried out at room temperature.

\section{Percoll density gradient centrifugation}

Centrifugation through $60 \%$ Percoll in Tyrode's solution was done following the method of Oshio (1988). For cauda epididymal spermatozoa, $1 \mathrm{ml}$ of sperm suspension was loaded on $9 \mathrm{ml}$ of $60 \%$ Percoll in modified Tyrode's solution without BSA. The Percoll solution was centrifuged at $30000 \mathrm{~g}$ for $30 \mathrm{~min}$ at $20^{\circ} \mathrm{C}$ with an angle rotor (Hitachi Type RP 65, Tokyo). Percoll formed a continuous density gradient as revealed with colour density marker beads. After centrifugation, the upper and lower fraction were collected from the top separately with a Pasteur pipette. Caput epididymal spermatozoa were also treated in a similar manner. After centrifugation, two distinct layers were obtained as in the case of cauda epididymal spermatozoa. The upper fraction, however, was heavily contaminated with other cell types, and only the lower fraction was used for lipid analysis. Percoll was not washed out from the collected sperm fractions. The number of spermatozoa was determined using a Thoma ruled haemocytometer.

\section{Lipid extraction and fractionation}

Lipids were extracted as described by Bligh and Dyer (1959) and stored at $-20^{\circ} \mathrm{C}$ until use. Lipids were fractionated on Silica gel 60 (Merck, Darmstadt) TLC plates. Total lipid and phospholipids were developed in chloroform:methanol: $\mathrm{H}_{2} \mathrm{O}$ $(65: 25: 4, \mathrm{v} / \mathrm{v})$ (Lapage, 1964), chloroform:methanol:28\% $\mathrm{NH}_{3}$ $(65: 25: 5, \mathrm{v} / \mathrm{v})$ (Synder, 1973), chloroform:acetone:methanol:acetic acid: $\mathrm{H}_{2} \mathrm{O}(6: 8: 2: 2: 1, \mathrm{v} / \mathrm{v})$ or chloroform:methanol:28\% $\mathrm{NH}_{3}$ $(65: 25: 5, \mathrm{v} / \mathrm{v})$ in the first dimension and chloroform:acetone: methanol:acetic acid: $\mathrm{H}_{2} \mathrm{O} \quad(6: 8: 2: 2: 1, \mathrm{v} / \mathrm{v})$ in the second dimension (Rouser et al., 1969). Neutral lipids were separated from phospholipids on TLC after development in chloroform: methanol: $\mathrm{H}_{2} \mathrm{O}(65: 25: 4, \mathrm{v} / \mathrm{v})$. Neutral lipids were further developed in petroleum ether:chloroform:acetone (4:1:1, v/v) (Jayard and Douce, 1977). The lipids were located by spraying with $0.02 \%(\mathrm{w} / \mathrm{v})$ Primurin solution in $80 \%$ acetone (Kooy and Kupers, 1979) and illumination with ultraviolet light. They were identified by their chromatographic mobilities relative to lipid standards and by specific reagents, i.e. molybdenum blue (Dittmer and Wells, 1964) for phospholipids, ninhydrin for amino lipids (Fahmy et al., 1961), anthrone for glycolipids (Yamakawa et al., 1960) and bismuthate for choline lipids (Beiss, 1964).

\section{Fatty acid analysis}

Lipid-bound fatty acids were analysed by GLC using a Shimadzu GC-8A (Shimadzu Seisakusho Ltd, Kyoto) with a hydrogen flame ionization detector. Chromatographic data were digitized and peak areas were integrated by a computer. The areas on the TLC plate corresponding to individual lipids except for sterols were scraped into screw cap test tubes and treated with $5 \% \mathrm{HCl}-$ methanol at $100^{\circ} \mathrm{C}$ for $3 \mathrm{~h}$. The resulting fatty acid methyl esters were extracted with hexane and analysed on a glass column $\left(3.2 \mathrm{~mm} \times 2.0 \mathrm{~m}, 210^{\circ} \mathrm{C}\right)$ packed with $5 \%$ Shinchrom E-71 on Shimalite AW. Major fatty acids were identified by comparison of their retention times and mass spectra with those of authentic methyl esters. The amounts of individual fatty acids were determined relative to docosaenoic acid (22:0) added as an internal standard. The total phospholipid and neutral lipid (except for sterol) were expressed as the amount of fatty acids measured by GLC.

\section{Sterol analysis}

Sterols were analysed as described previously (Awano et al., 1990). Sterols were separated on a column $(2.6 \mathrm{~mm} \times 2.0 \mathrm{~m})$ containing $2 \%$ OV-1 on Shimalite W (Shimadzu, Kyoto) operated at $260^{\circ} \mathrm{C}$. The amount of sterols in each sample was determined by comparing integrated peak areas with that of $5 \alpha$-cholestane added as an internal standard.

\section{Electron microscopy}

Before and after centrifugation, spermatozoa were fixed with $2.5 \%$ glutaraldehyde in $0.1 \mathrm{~mol}$ phosphate buffer $1^{-1}, \mathrm{pH} 7.4$, postfixed with $1 \% \mathrm{OsO}_{4}$, dehydrated in a series of ethanols, and embedded in Spurr for transmission electron microscopy (TEM). Downloaded from Bioscientifica.com at 04/26/2023 12:37:09PM 


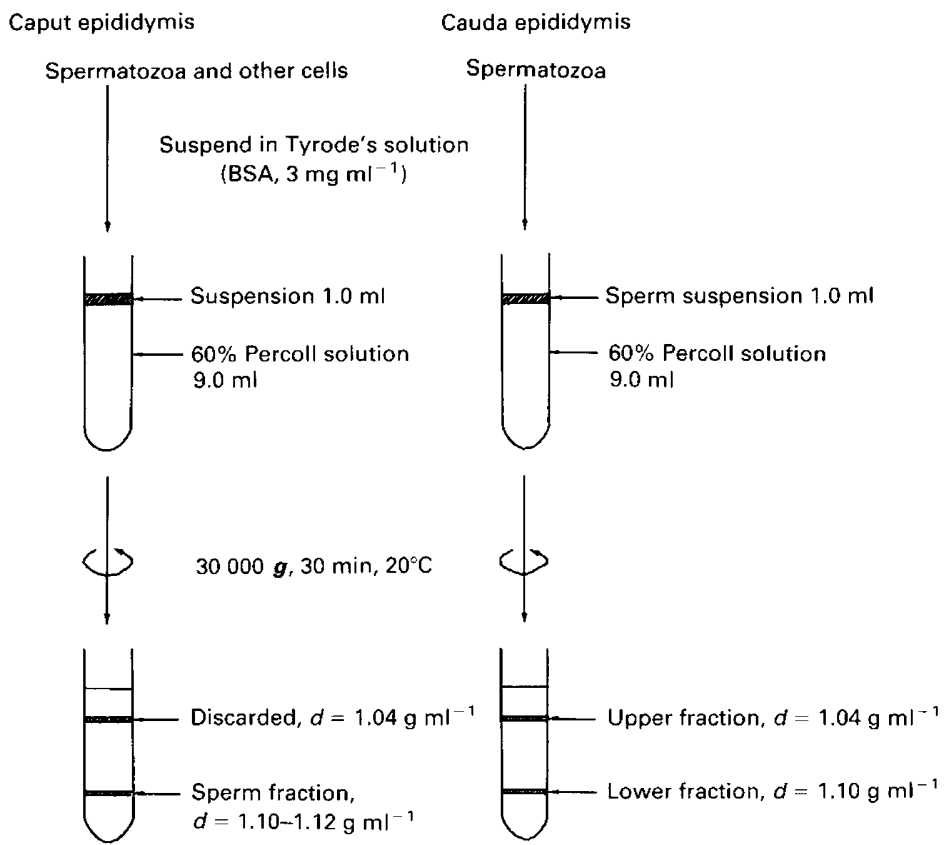

Fig. 1. Separation patterns of hamster caput and cauda epididymal spermatozoa.

Lead-citrate-stained sections were examined and photographed using a JOEL model JEM-100 B transmission electron microscope.

\section{Statistical analysis}

The percentage data were normalized by arc-sine transformation. Data were tested using Student's $t$ test.

\section{Results}

\section{Isolation of caput epididymal spermatozoa}

The sperm preparation was centrifuged through a linear Percoll density gradient to assess uniformity of the cells. Two distinct layers were obtained with densities (specific gravity) of $1.04 \mathrm{~g} \mathrm{~cm}^{-3}$ and $1.10-1.12 \mathrm{~g} \mathrm{~cm}^{-3}$, respectively (Fig. 1). The upper fraction contained mostly other cell types, whereas only spermatozoa were found in the lower fraction. This was confirmed by both phase-contrast microscopy and electron microscopy (Fig. 2). Electron microscopy revealed that the plasma and acrosomal membrane of caput epididymal spermatozoa were intact after Percoll density gradient centrifugation. The spermatozoa retained the cytoplasmic droplets. The caput epididymal spermatozoa isolated by this method were pure in comparison with unfractionated samples, so that this fraction of $1.10-1.12 \mathrm{~g} \mathrm{~cm}^{-3}$ was used to analyse the lipids of caput epididymal spermatozoa. However, the lipids of washed cauda epididymal spermatozoa were analysed without Percoll density gradient centrifugation, because there was no contamination by other cells.

\section{Lipid composition of caput and cauda epididymal spermatozoa}

Lipid compositions of the caput and the cauda epididymal spermatozoa were similar to each other. Phosphatidylcholine (PC), phosphatidylethanolamine (PE), diphosphatidylglycerol (DPG), sterol and neutral lipids were the main components. Sphingomyelin (SPM), lysophosphatidylcholine (LPC), phosphatidylinositol (PI) and phosphatidylserine (PS) were also detected. Fatty acid composition of purified caput epididymal spermatozoa and washed (unseparated) cauda epididymal spermatozoa are summarized (Table 1).

The fatty acid compositions of hamster caput and cauda epididymal spermatozoa were almost the same. No significant difference $(P>0.05)$ was observed in fatty acid composition between caput and cauda epididymal spermatozoa. The major fatty acids of hamster epididymal spermatozoa were palmitic acid (16:0), stearic acid (18:0), docosapentaenoic acid (22:5) and docosahexaenoic acid (22:6). The four fatty acids (16:0, 18:0, $22: 5,22: 6)$ comprised about $80 \%$ of the total fatty acid. Myristic acid (14:0), oleic acid (18:1), linoleic acid (18:2) and arachidonic acid $(20: 4)$ were also present. The long chain unsaturated fatty acids $(22: 5,22: 6)$ comprised approximately $40 \%$ of the total fatty acid. Both 22:5 and 22:6 represented higher percentages in hamster epididymal spermatozoa than in other mammalian spermatozoa. There was a tendency for higher percentages of longer fatty acids in the cauda epididymal than in the caput epididymal spermatozoa. No significant difference $(P>0.05)$ was observed in the amount of total fatty acid between the caput and cauda epididymal spermatozoa. The caput epididymal 

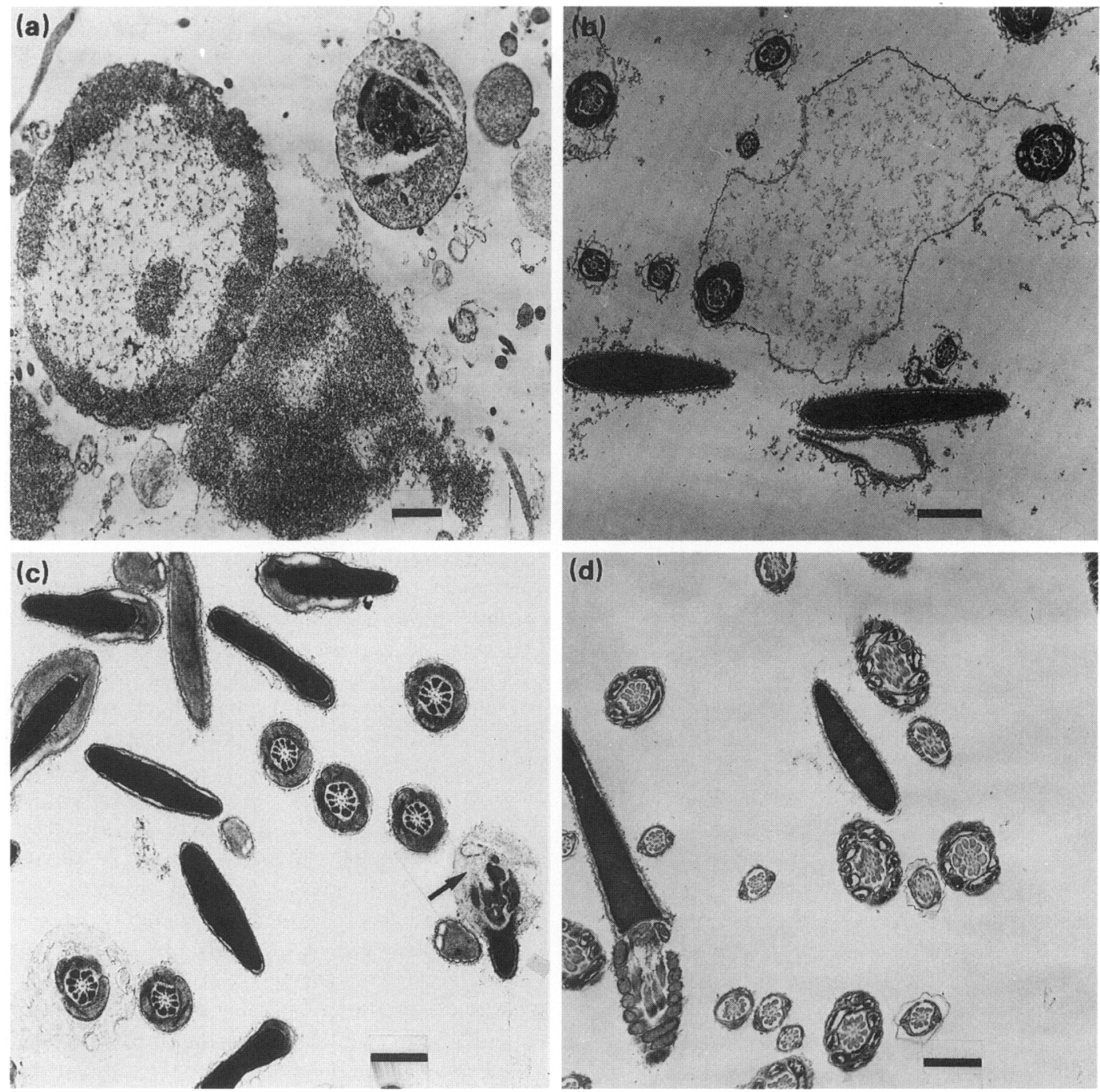

Fig. 2. Electron micrographs of hamster caput and cauda epididymal spermatozoa fractions obtained by Percoll density gradient centrifugation. (a) The upper fraction obtained from caput epididymal suspension; (b) the upper fraction of cauda epididymal spermatozoa; (c) the lower fraction of caput epididymal suspension; and (d) the lower fraction of cauda epididymal spermatozoa. Arrow indicates the cytoplasmic droplet. Scale bars $=1 \mu \mathrm{m}$.

spermatozoa contained $1.93 \pm 0.57 \mu \mathrm{mol} 10^{-8}$ spermatozoa of the total fatty acid, whereas the total fatty acid of the cauda epididymal spermatozoa was $1.75 \pm 0.31 \mu \mathrm{mol} 10^{-8}$ spermatozoa.

Sterols are the second major class of lipids in the sperm plasma membrane (Legault et al., 1979; Nikolopoulou et al., 1985). We analysed the sterol content and composition of hamster spermatozoa by GLC. As we reported previously (Awano et al., 1990), the sterol composition changed markedly during transit through the epididymis. Namely, the major sterol was cholesterol in the caput epididymal spermatozoa, whereas desmosterol and cholesta-7,24-dien-3 $\beta$-ol represented more than $90 \%$ of the total sterol in the cauda epididymal spermatozoa (Table 2). However, the amounts of the total sterol in the caput and cauda epididymal spermatozoa were the same within experimental error $(P>0.05)$.

\section{Lipid composition of upper and lower fractions of cauda epididymal spermatozoa}

Cauda epididymal spermatozoa were separated into two distinct layers after Percoll density gradient centrifugation (compare with Fig. 1). The lipids of both the upper and the lower fraction of cauda epididymal spermatozoa were analysed. The lower fraction with an apparent density of $1.12 \mathrm{~g} \mathrm{~cm}^{-3}$ consisted of morphologically normal spermatozoa, whereas the upper fraction with an apparent density of $1.04 \mathrm{~g} \mathrm{~cm}^{-3}$ contained morphologically abnormal spermatozoa, especially in the 
Table 1. Total fatty acid composition of hamster caput and cauda epididymal spermatozoa

\begin{tabular}{|c|c|c|}
\hline \multirow[b]{2}{*}{ Fatty acid } & \multicolumn{2}{|c|}{$\begin{array}{c}\text { Fatty acid composition } \\
\text { of epididymal spermatozoa (molar \%) }\end{array}$} \\
\hline & Caput & Cauda \\
\hline $14: 0$ & $4.3 \pm 1.7^{\mathrm{a}}$ & $3.3 \pm 1.6$ \\
\hline $16: 0$ & $30.7 \pm 10.2$ & $24.2 \pm 14.3$ \\
\hline $18: 0$ & $6.6 \pm 5.4$ & $11.5 \pm 6.8$ \\
\hline $18: 1$ & $6.4 \pm 1.9$ & $6.8 \pm 1.5$ \\
\hline $18: 2$ & $8.0 \pm 2.2$ & $8.8 \pm 0.8$ \\
\hline $20: 4$ & $3.5 \pm 1.4$ & $3.3 \pm 1.3$ \\
\hline $22: 5$ & $21.2 \pm 2.4$ & $22.8 \pm 1.1$ \\
\hline $22: 6$ & $17.3 \pm 3.0$ & $18.6 \pm 2.8$ \\
\hline
\end{tabular}

'Values are means \pm SD; $n=3$.

Table 2. Sterol composition of hamster caput and cauda epididymal spermatozoa

\begin{tabular}{lcr} 
& \multicolumn{2}{c}{$\begin{array}{c}\text { Sterol composition } \\
\text { (molar \%) }\end{array}$} \\
\cline { 2 - 3 } Sterols & Caput & Cauda \\
\hline Cholesterol & 99.9 & $7.1 \pm 0.5^{\mathrm{a}}$ \\
Desmosterol & Trace & $45.3 \pm 0.8$ \\
Cholesta-7,24-dien-33-ol & 0 & $47.6 \pm 0.6$ \\
\hline
\end{tabular}

${ }^{3}$ Values are means $\pm \mathrm{SD} ; n=9$

tail region. The lipid compositions of cauda epididymal spermatozoa are shown (Table 3). Total phospholipid and total neutral lipid are represented as the amounts of fatty acids. Phospholipids were the major class of lipid of the cauda epididymal spermatozoa. The amount of phospholipids presented here was consistent with the result of phosphorus analysis (data not shown). The spermatozoa of the upper fraction had a higher total fatty acid content than did those of the lower fraction $(P<0.05)$. The total fatty acid of the former fraction was about 1.4-fold that of the latter fraction. For some reason, the amount of total phospholipid after TLC separation exceeded the amount of total fatty acid in the upper fraction. The difference in the total sterol content, however, was not statistically significant. Total sterol: total phospholipid ratios were computed by the following equation: $\left\{\right.$ total sterol $\left(\mu \mathrm{mol} 10^{-8}\right.$ spermatozoa)/total phospholipid $\left(\mu \mathrm{mol} 10^{-8}\right.$ spermatozoa $\left.)\right\} \times 2$. The total sterol:total phospholipid ratios of the two fractions were different, because of the higher phospholipid content in the upper fraction. The ratio of the upper fraction was 0.11 , whereas the ratio of the lower fraction was 0.17 . The total sterol:total phospholipid ratio of 0.21 in hamster cauda epididymal spermatozoa (unseparated fraction) was considerably smaller than those in other mammalian spermatozoa such as bull, boar and rabbit (Davis, 1981).
The main components of total fatty acid were the same between the upper and the lower fraction (Table 4). Among them, however, the amounts of 18:2 and 22:5 in the lower fraction of cauda epididymal spermatozoa were higher than that of the upper fraction, whereas the amount of 18:0 in the former was lower than that in the latter $(P<0.05)$. Other fatty acids remained relatively constant.

Among phospholipids of cauda epididymal spermatozoa, PC was the most abundant followed by PE. PC and PE represented about $80 \%$ of the total phospholipid. DPG was less than $15 \%$ of the total phospholipid. PI, PS, SPM and LPC were present as minor components, comprising $3 \%$ of the total phospholipid (Table 5). As the major lipid class was phospholipid, we analysed the fatty acid composition of the seven major phospholipids in the two fractions of cauda epididymal spermatozoa. Four fatty acids (16:0, 18:0, 22:5, 22:6) comprised approximately $90 \%$ of the fatty acids in PC (Fig. 3). Long-chain unsaturated fatty acids, such as 22:5 and 22:6, were rather restricted to PC and PE. DPG was unique in that about $60 \%$ of the total fatty acid consisted of 18:2. SPM, LPC and PI plus PS were notable for their high 16:0 and 18:0 content. The fatty acid composition was different between PC and LPC. The composition of phospholipid-bound fatty acids was almost the same between the upper and the lower fraction (data not shown).

In addition to the major phospholipids, we also determined the composition of the free fatty acid pool, triacylglycerol (TG), 1,2-diacylglycerol (1,2-DG) and monoacylglycerol (MG) (Fig. 4). In these lipids, the contents of saturated fatty acids, especially 16:0 and 18:0, were high and long-chain unsaturated fatty acids, 22:5 and 22:6, were not observed.

Hamster cauda epididymal spermatozoa contained three main sterols which were identified by GC-MS spectrometry as cholesterol, desmosterol and cholesta-7,24-dien-3 $\beta$-ol. There was no difference in the sterol composition between the upper and the lower fraction of cauda epididymal spermatozoa (Table 6).

\section{Discussion}

Spermatozoa from hamster caput epididymides were purified by Percoll density gradient centrifugation. By this method, little contamination by other cells and cellular components was observed in the isolated spermatozoa. The isolated spermatozoa have almost the same density as that of the lower fraction $\left(1.12 \mathrm{~g} \mathrm{~cm}^{-3}\right.$ ) of cauda epididymal spermatozoa.

As reported by Awano et al. (1990), hamster spermatozoa show a marked change in the composition of sterols during the epididymal transit. The amount of cholesterol is much reduced with concomitant appearance of desmosterol and cholesta-7,24dien-3 $\beta$-ol. A few reports have been published concerning the composition of sterols in mammalian spermatozoa. In boar spermatozoa, an appearance of desmosterol, an intermediate of cholesterol synthesis, in the plasma membrane was observed during sperm maturation (Nikolopoulou et al., 1985). In hamster spermatozoa, the presence of desmosterol and its conversion to desmosterol sulfate were observed during sperm maturation (Legault et al., 1979). This would cause a change in surface charge of the spermatozoa. The increase in net negative charge Downloaded from Bioscientifica.com at 04/26/2023 12:37: ๑9PM 
Table 3. Lipid content of hamster cauda epididymal spermatozoa

\begin{tabular}{lccc}
\hline & \multicolumn{3}{c}{ Lipid content $\left(\mu \mathrm{mol} 10^{-8}\right.$ spermatozoa) } \\
\cline { 2 - 4 } Lipid & Upper fraction & Lower fraction & Unseparated \\
\hline $\begin{array}{l}\text { Total fatty acid } \\
\text { Total phospholipid }\end{array}$ & $2.21 \pm 0.11^{\mathrm{a}}$ & $1.53 \pm 0.08$ & $1.75 \pm 0.31$ \\
$\begin{array}{c}\text { Total neutral lipid } \\
\text { (except for sterols) }\end{array}$ & $3.14 \pm 0.04$ & $1.49 \pm 0.17$ & $1.45 \pm 0.54$ \\
Total sterol & $0.29 \pm 0.16$ & $0.10 \pm 0.02$ & $0.11 \pm 0.01$ \\
& $0.17 \pm 0.05$ & $0.13 \pm 0.04$ & $0.15 \pm 0.03$ \\
\hline
\end{tabular}

'Values are means $\pm \mathrm{SD} ; n=3$.

${ }^{b}$ The amount of fatty acids bound to phospholipids.

'The amount of fatty acids bound to neutral lipids.

Table 4. Fatty acid composition of hamster cauda epididymal spermatozoa

\begin{tabular}{|c|c|c|c|}
\hline \multirow{2}{*}{$\begin{array}{l}\text { Fatty } \\
\text { acid }\end{array}$} & \multicolumn{3}{|c|}{ Fatty acid composition (molar \%) } \\
\hline & Upper fraction & Lower fraction & Unseparated \\
\hline $14: 0$ & $3.1 \pm 0.3^{\mathrm{a}}$ & $4.5 \pm 2.0$ & $3.2 \pm 1.6$ \\
\hline $16: 0$ & $18.4 \pm 0.3$ & $18.7 \pm 1.1$ & $24.3 \pm 12.0$ \\
\hline $18: 0$ & $21.2 \pm 2.7$ & $14.5 \pm 0.9$ & $10.9 \pm 7.0$ \\
\hline $18: 1$ & $8.2 \pm 0.6$ & $7.2 \pm 0.3$ & $6.7 \pm 1.5$ \\
\hline $18: 2$ & $4.7 \pm 0.7$ & $9.2 \pm 0.3$ & $8.1 \pm 0.7$ \\
\hline $20: 4$ & $3.2 \pm 1.4$ & $2.9 \pm 0.1$ & $3.1 \pm 1.3$ \\
\hline $22: 5$ & $18.9 \pm 0.9$ & $21.9 \pm 1.2$ & $22.5 \pm$ \\
\hline $22: 6$ & $16.5 \pm 1.0$ & $19.6 \pm 2.5$ & $18.4 \pm 2.8$ \\
\hline
\end{tabular}

${ }^{2}$ Values are means $\pm \mathrm{SD} ; n=3$.

Table 5. Composition of phospholipids of hamster cauda epididymal spermatozoa

Phospholipid composition (molar \%)

Phospholipids Upper fraction Lower fraction Unseparated

\begin{tabular}{lrrr}
\hline PC & 58.6 & 53.0 & 54.3 \\
PE & 27.1 & 30.2 & 29.7 \\
DPG & 11.5 & 14.1 & 13.7 \\
PI+PS & 1.3 & 1.3 & 1.1 \\
SPM & 0.6 & 0.7 & 0.6 \\
LPC & 1.0 & 0.7 & 0.6 \\
\hline
\end{tabular}

PC: phosphatidylcholine; PE: phosphatidylethanolamine; DPG: diphosphatidylglycerol; PI: phosphatidylinositol; PS: phosphatidylserine; SPM: sphingomyelin; LPC: lysophosphatidylcholine.

at the lipid-water interface of the plasma membrane observed during maturation could be caused at least in part by an increase in the content of sterol sulfate (Yanagimachi et al., 1972). It appears that some of the enzymes responsible for cholesterol synthesis, such as desmosterol reductase and $\Delta^{24}$-reductase, are not present or are not activated in hamster cauda epididymis, causing the accumulation of intermediates. The removal of cholesterol from the plasma membrane was found to be associated with capacitation and acrosome reaction in rat spermatozoa (Davis, 1981), and the time required for capacitation was considered to correlate with the sperm cholesterol:phospholipid molar ratio of the spermatozoa (Davis, 1980). Hamster spermatozoa have a relatively lower cholesterol:phospholipid molar ratio as compared with the time for capacitation.

In hamster spermatozoa, phospholipid is the major lipid. The specific function of this lipid in biological membranes is not yet well understood, although it has been suggested that phospholipids may serve as endogenous sources of metabolic fuel for spermatozoa in the epididymis (Scott and Dawson, 1968). The fatty acid compositions of hamster caput and cauda epididymal spermatozoa as well as that of the major phospholipids were found to be dominated by 16:0, 22:5 and 22:6. A relatively large amount of stearic acid (18:0) was also present. The phospholipid-bound fatty acids of hamster spermatozoa contain higher amounts of polyunsaturated fatty acids such as $22: 5$ and 22:6 than do other mammalian spermatozoa (Jain and Anand, 1976; Darin-Bennet et al., 1977; Parks and Lynch, 1992; Poulos et al., 1973a). The difference in phospholipid-bound fatty acid composition between PC and LPC may be due to the fact that phospholipase $A_{2}$ specifically cleaves the acyl ester (polyunsaturated fatty acids) at position 2 of the glycerol moiety of PC.

Several investigators reported that the lipid content of spermatozoa decreases during epididymal maturation in rams (Evans and Setchell, 1979b) and rats (Adams and Johnson, 1977). In the present study, however, the amounts of total fatty acid and total sterol did not change during transit through the epididymis in hamster spermatozoa. These results indicate that a large alteration in the lipid of hamster spermatozoa does not occur during epididymal maturation. The discrepancy could be attributed to species differences. However, contamination by other cells into caput epididymal sperm fraction, which was excluded in the present experiment, should be considered in the cases of other species.

Oshio (1988) reported that two separated fractions were obtained by Percoll density gradient centrifugation in hamster cauda epididymal spermatozoa. There were immature sperma- 

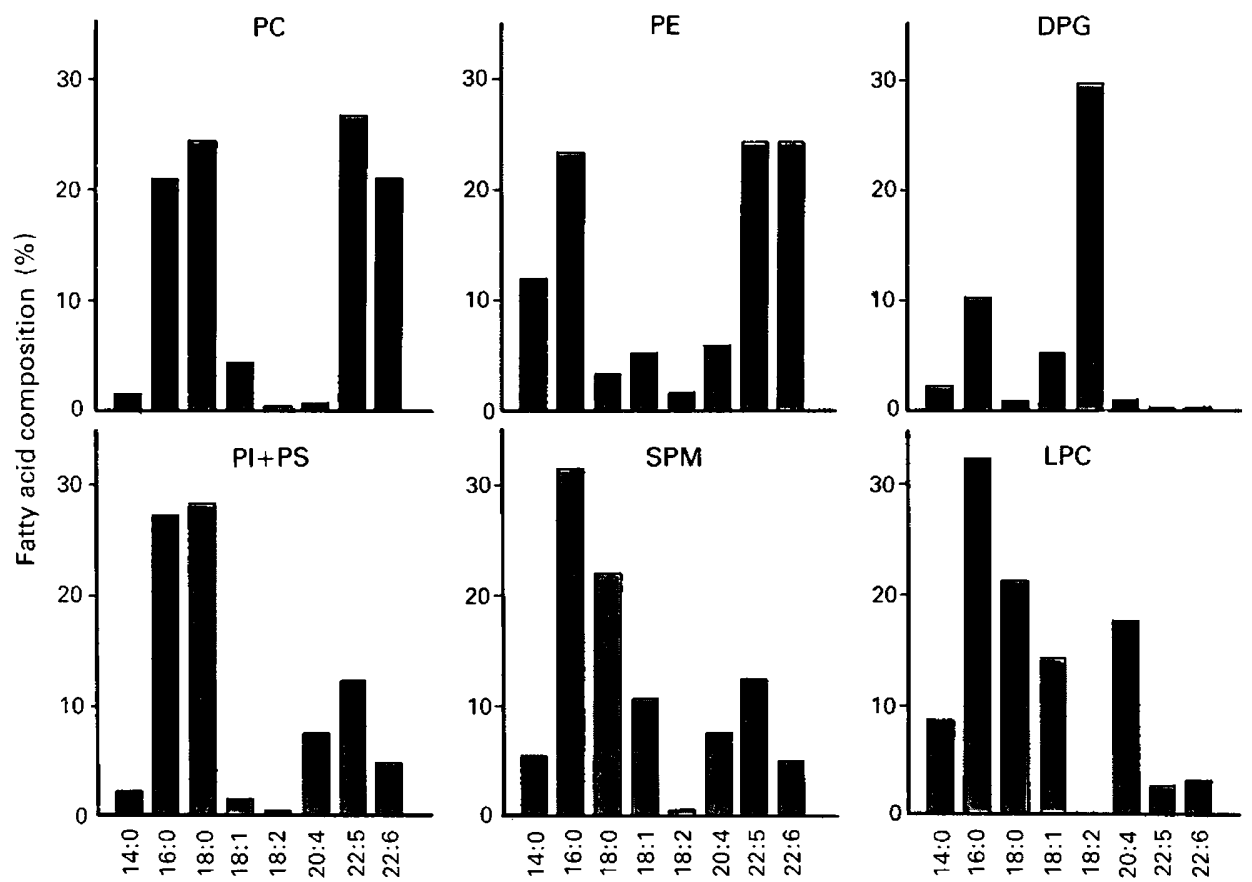

Fig. 3. Fatty acid composition of phospholipids of hamster cauda epididymal spermatozoa. PC: phosphatidylcholine; PE: phosphatidylethanolamine; DPG: diphosphatidylglycerol; PI: phosphatidylinositol; PS: phosphatidylserine; SPM: sphingomyelin; LPC: lysophosphatidylcholine.
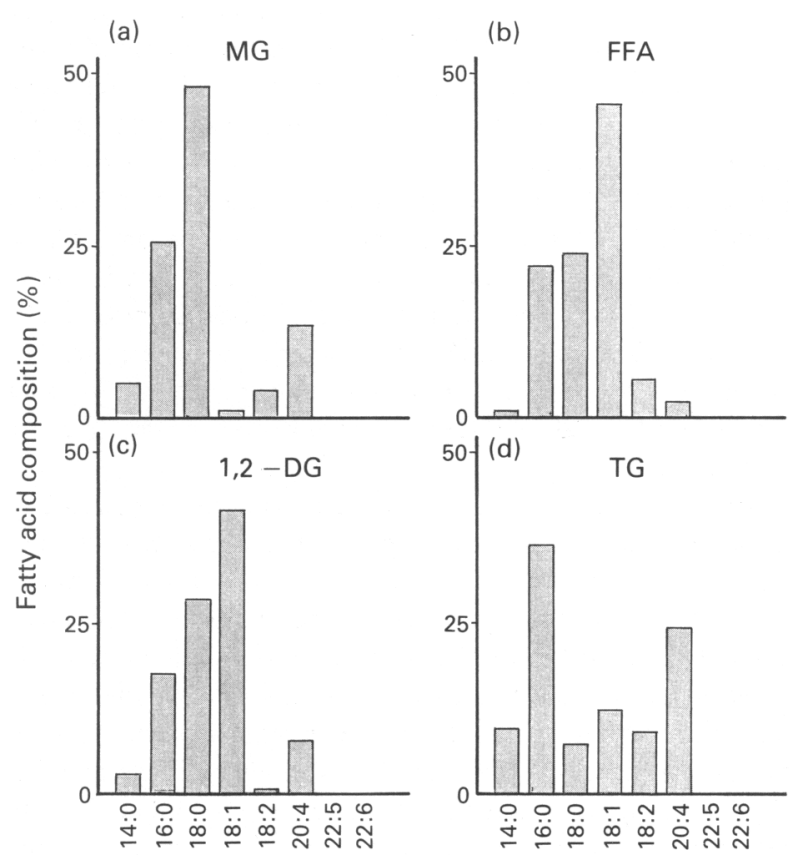

Fig. 4. Fatty acid composition of neutral lipids (except for sterols) of hamster cauda epididymal spermatozoa. (a) MG: monoacylglycerol; (b) FFA: free fatty acids; (c) 1,2-DG: 1,2-diacylglycerol; and (d) TG: triacylglycerol. tozoa in the upper fraction, and mature and motile spermatozoa in the lower fraction. In the present experiment, the total fatty acid content of the upper fraction of cauda epididymal spermatozoa was higher, 1.4-fold, than that of the lower fraction. The difference is mainly derived from the phospholipid content. After the release of spermatozoa from the testis, the cytoplasmic droplet migrates along the midpiece during epididymal transit (Austin, 1985), and hamster cauda epididymal spermatozoa still have the cytoplasmic droplet on the distal end of the midpiece near the tail (Hirao and Hiraoka, 1986). The cytoplasmic droplet contains phospholipids. Phospholipid compositions of the cytoplasmic droplets and spermatozoa were reported to be quite similar (Anand and Atreja, 1986), and the cytoplasmic droplets separated from goat epididymal spermatozoa contain $31 \%$ of the total lipid present in caput sperm cells. At least some of the observed differences in the contents of the total fatty acid and phospholipids of the two fractions obtained with hamster cauda epididymal spermatozoa can be attributed to the decrease of the cytoplasmic droplet. However, the difference in density cannot be explained by the lipid content, especially the content of phospholipids only. There are lipids in the plasma membrane, acrosomal membrane, mitochondrial membrane and cytosolic fraction of spermatozoa. It is not clear which is the location of increased lipids in the spermatozoa contained in the upper fraction. If hamster cauda epididymal spermatozoa were demembranated with Triton X-100, they showed a single band, of density $1.10 \mathrm{~g} \mathrm{~cm}^{-3}$, after Percoll density gradient centrifugation (data not shown). This result suggests that the plasma membrane has some correlation with the observed difference in density of the two fractions, although it is not 
Table 6. Sterol composition of hamster cauda epididymal spermatozoa

\begin{tabular}{|c|c|c|c|}
\hline \multirow[b]{2}{*}{ Sterols } & \multicolumn{2}{|c|}{ Sterol composition (molar \%) } & \multirow[b]{2}{*}{ Unseparated } \\
\hline & Upper fraction & Lower fraction & \\
\hline Cholesterol & $8.6 \pm 1.1^{\mathrm{a}}$ & $7.8 \pm 1.8$ & $7.1 \pm 0.7$ \\
\hline Desmosterol & $44.9 \pm 0.8$ & $46.2 \pm 0.7$ & $45.3 \pm 1.4$ \\
\hline Cholesta-7,24-dien-3 $\beta$-ol & $48.2 \pm 3.1$ & $45.7 \pm 2.3$ & $47.0 \pm 0.7$ \\
\hline
\end{tabular}

${ }^{2}$ Values are means $\pm \mathrm{SD} ; n=5$.

certain whether the difference is due to the degree of sperm maturation.

The authors thank M. Morisaki, Kyoritsu College of Pharmacy, for useful suggestions of sterol analysis and N. Morisaki, Institute of Applied Microbiology, the University of Tokyo, for her advice. We are indebted to $T$. Mohri for EM analysis.

\section{References}

Adams CS and Johnson AD (1977) The lipid content of epididymal spermatozoa of Rattus norvegicus Comparative Biochemistry and Physiology 58B 409-411

Amann RP, Hay SR and Hammerstedt RH (1982) Yield, characteristics, motility and cAMP content of sperm isolated from seven regions of ram epididymis Biology of Reproduction 27 723-733

Anand SR and Atreja SK (1986) Lipids in goat cytoplasmic droplets and spermatozoa in transit from the caput to the cauda epididymis Indian Journal of Biochemistry and Biophysics 23 258-265

Atherton RW, Khatoon S, Schoff PK and Haley BE (1985) A study of rat epididymal sperm adenosine $3^{\prime}, 5^{\prime}$-monophosphate-dependent protein kinase: maturation differences and cellular location Biology of Reproduction 32 155-171

Austin CR (1985) Sperm maturation in the male and female genital tracts Biology of Fertilization Vol 2 pp 121-155 Eds CB Metz and A Monroy. Academic Press, New York

Awano M, Kawaguchi A, Morisaki M and Mohri H (1990) Identification of cholesta-7,24-dien-3 $\beta$-ol and desmosterol in hamster cauda epididymal spermatozoa Lipids 24 662-664

Bavister BD (1969) Environmental factors important for in vitro fertilization in the hamster Joumal of Reproduction and Fertility 18 544-545

Bedford JM (1963) Changes in the electrophoretic properties of rabbit spermatozoa during passage through the epididymis Nature 200 1178-1180

Beiss U (1964) Zur papierchromatographischen Auftrennung von Pflanzenlipiden Journal of Chromatography 13 104-110

Bligh DG and Dyer WJ (1959) A rapid method of total lipid extraction and purification Canadian Journal of Biochemistry and Physiology 37 911-917

Cooper GW and Bedford JM (1971) Acquisition of surface charge by the plasma membrane of mammalian spermatozoa during epididymal maturation Anatomical Record 169 300-301

Cornwall GA, Vreeburg JT, Holland MK and Orgebin-Crist M (1990) Interactions of labeled epididymal secretory proteins with spermatozoa after injection of ${ }^{35} \mathrm{~S}$-methionine in the mouse Biology of Reproduction 43 121-129

Dacheux JL, Dacheux F and Paquignon M (1989) Changes in sperm surface membrane and luminal protein fluid content during epididymal transit in the boar Biology of Reproduction 40 635-651

Darin-Bennet A, White IG and Hoskins DD (1977) Phospholipids and phospholipid-bound fatty acids and aldehydes of spermatozoa and seminal plasma of rhesus monkeys Journal of Reproduction and Fertility 49 119-122

Davis BK (1980) Interaction of lipids with the plasma membrane of sperm cells. I. The antifertilization action of cholesterol Archives of Andrology 5 249-254

Davis BK (1981) Timing of fertilization in mammals: sperm cholesterol/ phospholipid ratio as a determinant of the capacitation interval Proceedings of National Academy of Sciences, USA 8 7560-7564
Dittmer JD and Wells MA (1964) A simple, specific spray for the detection of phospholipids on thin-layer chromatograms Joumal of Lipid Research 5 126-127

Evans RW and Setchell BP (1979a) Lipid changes in boar spermatozoa during maturation, with some observations on the flow and composition of boar rete testis fluid Joumal of Reproduction and Fertility 57 189-196

Evans RW and Setchell BP (1979b) Lipid changes during epididymal maturation in ram spermatozoa collected at different times of the year journal of Reproduction and Fertility 57 197-203

Fahmy AR, Niederwieser A, Pataki G and Brenner M (1961) DünnschichtChromatographie von Aminosäuren auf Kieselgel G. Eine Schnellmethode zur Trennung und zum qualitativ en Nachweis von 22 Aminosäuren Helvetica Chimica Acta 44 2022-2026

Hammerstedt RH, Keith AD, Hay SR, Deluca N and Amann RP (1979) Changes in ram sperm membrane during epididymal transit Archives of Biochemistry and Biophysics 196 7-12

Hirao U and Hiraoka J (1986) Morphological characteristics of the cytoplasmic droplet of hamster spermatozoon at various stages after spermiation Wakayama Medical Reports 29 9-19

Jain YC and Anand SR (1976) Fatty acids and fatty aldehydes of buffalo plasma and sperm lipid Joumal of Reproduction and Fertility 47 261-267

Jayard J and Douce R (1977) Site of synthesis of phosphatidic acid and diacylglycerol in spinach chloroplasts Biochimica et Biophysica Acta $\mathbf{4 8 6}$ 273-285

Kooy D and Kupers HGJM (1979) Fluorescent retrograde double labeling: axonal branching in the ascending raphe and nigral projections Science 204 873-875

Lapage M (1964) Isolation and characterization of an esterified form of steryl glucoside Journal of Lipid Research 5 587-592

Lavon U, Volcani R, Amir D and Danan D (1966) The specific gravity of bull spermatozoa from different parts of the reproductive tract Journal of Reproduction and Fertility 12 597-599

Legault Y, Bouthillier G, Bleau G, Chapdelaine A and Roberts KD (1979) The sterol and sterol sulfate content of the male hamster reproductive tract Biology of Reproduction 20 1213-1219

Lui HW, Wang CF and Muller C (1991) Identification of two maturation-related, wheat-germ-lectin-binding proteins on the surface of mouse sperm Acta Anatomica 142 165-170

Magargee SF, Kunze E and Hammerstedt RH (1988) Changes in lectin-binding features of ram sperm surfaces associated with epididymal maturation and ejaculation Biology of Reproduction 38 667-685

Nikolopoulou M, Soucek DA and Vary JC (1985) Changes in the lipid content of boar sperm plasma membranes during epididymal maturation Biochimica ef Biophysica Acta 815 486-498

Oshio S (1988) Apparent densities of spermatozoa of various mammalian spermatozoa Gamete Research 20 159-164

Parks JE and Lynch DV (1992) Lipid composition and thermotropic phase behavior of boar, bull, stallion, and rooster sperm membranes Cryobiology 29 255-266

Poulos A, Darin-Bennett A and White IG (1973a) The phospholipid-bound fatty acids and aldehydes of mammalian spermatozoa Comparative Biochemistry and Physiology 46B 541-549

Poulos A, VogImayr JK and White IG (1973b) Phospholipid changes in spermatozoa during passage through the genital tract of the bull Biochimica et Biophysica Acta 306 194-202

Poulos A, Brown-Woodman PDC, White IG and Cox RI (1975) Changes in phospholipids of ram spermatozoa during migration through the epididymis Downloaded from Bioscientifica.com at 04/26/2023 12:37: 09PM 
and possible origin of prostaglandin $F_{2 a}$ in testicular and epididymis fluid Biochimica et Biophysica Acta 388 12-18

Rodriguez-Martinez H, Courtens JL, Kvist U and Plöen L (1990) Immunocytochemical localization of nuclear protamine in boar spermatozoa during epididymal transit Joumal of Reproduction and Fertility 89 591-595

Rouser G, Kritchevsky G, Yamamoto A, Simon G, Galli C and Bauman J (1969) Diethylaminoethyl and triethylaminoethyl cellulose column chromatographic procedures for phospholipids, glycolipids, and pigments. Methods in Enzymology Vol 14 pp 272-317 Ed. JM Lowenstein. Academic Press, New York

Schlegel RA, Hammerstedt RH, Cofer GP, Kozarsky K, Freidus D and Williamson P (1986) Changes in the organization of the lipid bilayer of the plasma membrane during spermatogenesis and epididymal maturation Biology of Reproduction 34 379-391

Scott TW and Dawson RMC (1968) Metabolism of phospholipid by spermatozoa and seminal plasma Biochemical journal 108 457-463

Synder F (1973) Thin-layer chromatographic behavior of glycerolipid analogue containing ether, ester, hydroxyl, and ketone groupings Journal of Chromatography 82 7-14

Teichman RJ, Cummins IM and Takei GH (1974) The characterization of a Malachite Green-stainable glutaraldehyde-extractable phospholipid in rabbit spermatozoa Biology of Reproduction 10 565-577
Vijayasarathy JK, Keith AD, Hay S, Deluca N and Amann RP (1979) Regional differentiation in bull sperm plasma membranes Biochemical and Biophysical Research Communications 108 760-764

White CR and Aitken RJ (1989) Influence of epididymal maturation on cyclic AMP levels in hamster spermatozoa International Joumal of Andrology 12 29-43

Wolf DE and Voglmayr JK (1984) Diffusion and regionalization in membrane of maturing ram spermatozoa Journal of Cell Biology 98 1678-1684

Wooten MW, Voglmayr JK and Wrenn RW (1986) Characterization of cAMPdependent protein kinase and its endogenous substrate proteins in ram testicular, cauda epididymal, and ejaculated spermatozoa Gamete Research 16 $57-68$

Yamakawa T, Irie R and Iwanaga M (1960) The chemistry of lipid of posthemolytic residue or stroma of erythrocyte. IX. Silicic acid chromatography of mammalian stroma glycolipids Joumal of Biochemistry 48 490-507

Yanagimachi R, Noda YD, Fugimoto M and Nicolson GL (1972) The distribution of negative surface charges on mammalian spermatozoa American Journal of Anatomy 135 497-520 